\title{
Robot Approaching and Engaging People in a Human-Robot Companion Framework
}

\author{
Ely Repiso
}

\author{
Anaís Garrell
}

Alberto Sanfeliu

\begin{abstract}
This paper presents a new model to make robots capable of approaching and engaging people with a human-like behavior, while they are walking in a side-by-side formation with a person. This method extends our previous work [1], which allows the robot to adapt its navigation behaviour according to the person being accompanied and the dynamic environment. In the current work, the robot is able to predict the best encounter point between the human-robot group and the approached person. Then, in the encounter point the robot modifies its position to achieve an engagement with both people. The encounter point is computed using a gradient descent method that takes into account all people predictions. Moreover, we make use of the Extended Social Force Model (ESFM), and it is modified to include the dynamic goal. The method has been validated over several situations and in real-life experiments, in addition, a user study has been realized to reveal the social acceptability of the robot in this task.
\end{abstract}

\section{INTRODUCTION}

Lately, robots are moving from industry and laboratories to the real world. Therefore, it is necessary to investigate deeply in human-robot interactive and collaborative tasks, some of these could be: accompany people [2], approach people [3], [4], or recognize if people are interested in interacting with them [5], [6].

In urban environments, humans tend to approach to other pedestrians to interact with them. If people navigate in groups and want to interact with other person outside the formation, they have to re-organize their configuration to interact comfortably.

Approaching other people is a natural task for humans, however, for companion robots it is a complex assignment. Robot has to understand and adapt its behaviour to both people, the accompanied person and the approached one. On the one hand, the robot has to accompany a person in a human-like way. And, on the other hand, the robot has to predict the best encounter point as humans do.

Work supported by the Spanish Ministry of Science and Innovation under project ColRobTransp (DPI2016-78957-RAEI/FEDER EU), RoboCom++ (FLAG-ERA JCT 2016) and by the Spanish State Research Agency through the Mara de Maeztu Seal of Excellence to IRI (MDM-2016-0656). Ely Repiso is also suported by Spanish Ministry of Science and Innovation under a FPI-grant, BES-2014-067713.

The authors are with the Institut de Robòtica i Informàtica Industrial (CSIC-UPC). Llorens Artigas 4-6, 08028 Barcelona, Spain. \{erepiso, agarrell, sanfeliu\}@iri.upc.edu

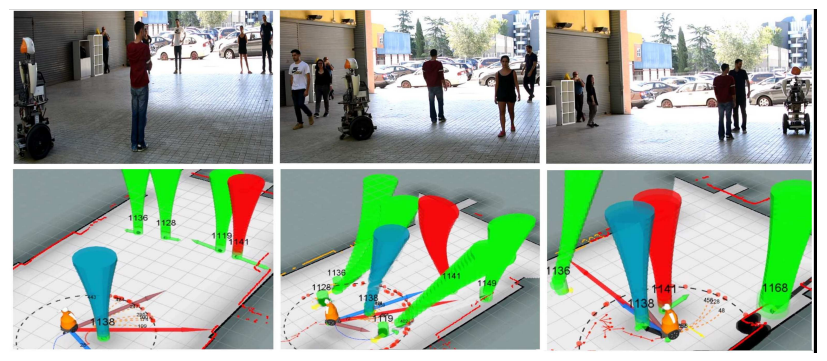

Fig. 1: Real-life experiments in FME. The robot avoids several pedestrians in the environment while accompanying a person and approaches another human.

Fig. 1 represents different moments of the real-life experiments. Here, the robot has to carry out some complex sub-tasks: anticipate the people's movements, deal with uncertainties like people occlusions, interaction with several pedestrians, prediction and autonomous navigation.

One of the objectives of social robots when they collaborate with people, is to follow similar social norms as humans. Some researchers have worked in the proxemic rules between humans [7], and in the relations between humans and robots, [8]. Furthermore, if the robot has to approach people, it must know when [9] and how [4] to approach humans.

Regarding the researches that use the proxemic rules between humans and robots, the work [10] investigated how the personality affects the spatial zones. Syrdal et al [8] studied the person-robot distances in different interactions: verbal, physical and no interaction. Some studies [11] found that people prefer the robot to approach by one of its sides.

Other approaches inferred people behavior and selected only the person interested to interact with the robot. In [6], authors evaluated a new approach to deduce through the trajectory of a person if he/she was interested in interact. Also, [12] implemented an approaching robot's behaviour to select people interested in interact according to their positions. These approaches could be applied in real-life applications, as in [13], where the authors deduced the interest of the person to obtain robot information by its proximity to the robot. Finally, Kato et al. [9] implemented a robot proactively behaviour to help the visitors only when they need it.

Finally, the work of [4] focused on developing robot strategies to approach moving persons, but their approach obtained a linear prediction that did not consider the dynamic and static obstacles. 
In contrast to previous approaches, we use the ESFM to get people's predictions and the robot's navigation, thus, the robot obtains human-like behaviors. Also, the robot has to develop two complex tasks at the same time: approaching and accompanying a person, which increases the difficulty for the robot. Furthermore, the robot predicts the best encounter position as humans do, taking into account the trajectories of the group and the approached person.

In the remainder of the paper, Sec. II introduces the implemented approach. The metrics of performances are explained in Sec. III. Sec. IV describes the results of synthetic experiments, real-life experiments, and the user study. Finally, conclusions are given in Sec. V.

\section{METHOD}

This section explains the approaching and the engagement tasks, while the robot is accompanying another person. We assume that the person of the group knows the target person (it is the person to be approached by the group). The accompany task follows the same procedure that was explained in our previous work [14].

\section{A. The Approaching Method}

In previous work [1], we implemented a simple strategy for the pair human-robot to approach the target person. In this paper, we present a new approach which optimally achieve the tasks of approaching and engagement.

This section is divided in two parts which are the steps of the approaching method: approaching to the dynamic goal and proactive configuration to create an engagement.

1) Stage 1. Approaching to the Dynamic Goal: Our goal is to make the robot capable of accompanying a person until his/her destination, $\mathcal{D}_{n}^{f}$, while approaches a moving target, $\mathcal{D}_{n}^{d g}$, to do it, we use the resultant force of Eq. 1 to control the movement of the robot. Where $n$ means the actual discrete time.

$$
\mathbf{F}_{r}=\alpha \mathbf{f}_{r, d}^{\text {goal }}\left(\mathcal{D}_{n}^{d g}\right)+\beta \mathbf{f}_{r, p}^{\text {goal }}\left(\mathcal{D}_{n}^{f}\right)+\left(\gamma \mathbf{F}_{r}^{p e r}+\delta \mathbf{F}_{r}^{\text {obs }}\right)
$$

The parameters $\{\alpha, \beta, \gamma, \delta\}$ were obtained from [15] and satify: $1=\alpha+\beta+\gamma+\delta$.

This force is composed of four sub-forces: $\mathbf{f}_{r, d}^{\text {goal }}\left(\mathcal{D}_{n}^{d g}\right)$, the attractive force exerted by the dynamic goal $\mathbf{f}_{r, p}^{\text {goal }}\left(\mathcal{D}_{n}^{f}\right)$, the attractive force to maintain the formation between the robot and the accompanied person $\mathbf{F}_{r}{ }^{\text {per }}$, the repulsive force respect to pedestrians of the environment, and $\mathbf{F}_{r}{ }^{\text {obs }}$ the repulsive force respect to obstacles.

$\mathbf{F}_{r}{ }^{\text {per }}$ allows the robot to avoid unnecessary interactions with people who are not the accompanied person, or the approached person. This force was defined in a previous work [16].

This repulsive force depends on the distance between the robot and the pedestrian. To calculate this distance we need to consider all the positions of the pedestrians in the environment, at each instant of time. Then, to obtain the propagated positions of all people we use Alg. 2 and the forces of the lines 7 and 8 are obtained from Eq. 2. The Alg. 2 calculates the prediction of any moving entity, for example a pedestrian.

$$
\mathbf{F}_{p_{j}}=\alpha \mathbf{f}_{p_{j}, d}^{\text {goal }}\left(\mathcal{D}_{n}^{g}\right)+\left(\gamma \mathbf{F}_{p_{j}}^{\text {per }}+\delta \mathbf{F}_{p_{j}}^{o b s}\right) .
$$

$F_{p_{j}}$ is the resultant force to move the person at each instant of time to the person's final goal. This force is a combination of three sub-forces: an attractive force exerted by person goal $\mathbf{f}_{p_{j}, d}^{\text {goal }}\left(\mathcal{D}_{n}{ }^{g}\right)$ and two repulsive forces $\mathbf{F}_{p_{j}}^{\text {per }}$ and $\mathbf{F}_{p_{j}}{ }^{\text {obs }}$ due to other people and obstacles.

The parameters values satisfy $1=\alpha+\gamma+\delta$ and were obtained from [15]. The attractive force exerted by the person's final goal, $\mathbf{f}_{p_{j}, d}^{\text {goal }}\left(\mathcal{D}_{n}{ }^{g}\right)$ is analog to the attractive force exerted by robot's final goal, $\mathbf{f}_{r, d}^{\text {goal }}\left(\mathcal{D}_{n}^{d g}\right)$. The repulsive forces respect to pedestrians and obstacles are analog to the repulsive forces of the robot.

The repulsive force of the robot respect to the obstacles in the environment is $\mathbf{F}_{r}^{o b s}$. This force was defined in a previous work [15]. These repulsive forces are used by the robot to avoid possible collisions with the obstacles.

The attractive force to maintain the formation between the robot and the accompanied person, $\mathbf{f}_{r, p}^{\text {goal }}\left(\mathcal{D}_{n}^{f}\right)$, introduced in our previous work [14], has been split in two forces, the first one is $\mathbf{f}_{r, d}^{\text {goal }}\left(\mathcal{D}_{n}^{d g}\right)$ which manages the dynamic goal and the second one is $\mathbf{f}_{r, p}^{\text {goal }}\left(\mathcal{D}_{n}^{f}\right)$ which manages the formation of the group.

The attractive force exerted by the dynamic goal, $\mathbf{f}_{r, d}^{\text {goal }}\left(\mathcal{D}_{n}^{d g}\right)$, pushes the robot to approach the moving target person. This force assumes that the robot tries to adapt its velocity within a relaxation time $k^{-1}$, but taking into account at the same time that the final destination is a dynamic goal, $\mathcal{D}_{n}^{d g}$, which is computed by an optimization process. The definition of this force is:

$$
\mathbf{f}_{r, d}^{\text {goal }}\left(\mathcal{D}_{n}^{d g}\right)=k\left(\mathbf{v}_{r}^{0}\left(\mathcal{D}_{n}^{d g}\right)-\mathbf{v}_{r}\right)
$$

where, $\mathbf{v}_{r}^{0}\left(\mathcal{D}_{n}^{d g}\right)$ is the desired velocity vector to reach the dynamic goal $\mathcal{D}_{n}^{d g}$.

Moreover, the robot has to compute the dynamic goal, $\mathcal{D}_{n}^{d g}=\left(x^{d g}, y^{d g}\right)$, where the group encounters the target person. This goal is optimally computed using a gradient descent method, see Alg. 1, which obtains the destination that minimizes the time until get-together. In combination with the Anticipative Kinodynamic Planer to Accompany People (AKP-AP), the method also optimizes the traveled distance, the effort of accomplishing the companion task and the effort to avoid obstacles and pedestrians.

Alg. 1, computes $t_{n}$, which is the minimum encounter time . The method has to propagate the positions (using the Alg 2) of all people. In each iteration, the propagation of the approached person is used as dynamic goal to obtain the estimated group's position. Finally, when the termination condition is fulfilled, the dynamic goal and the minimum time to approach are obtained. 


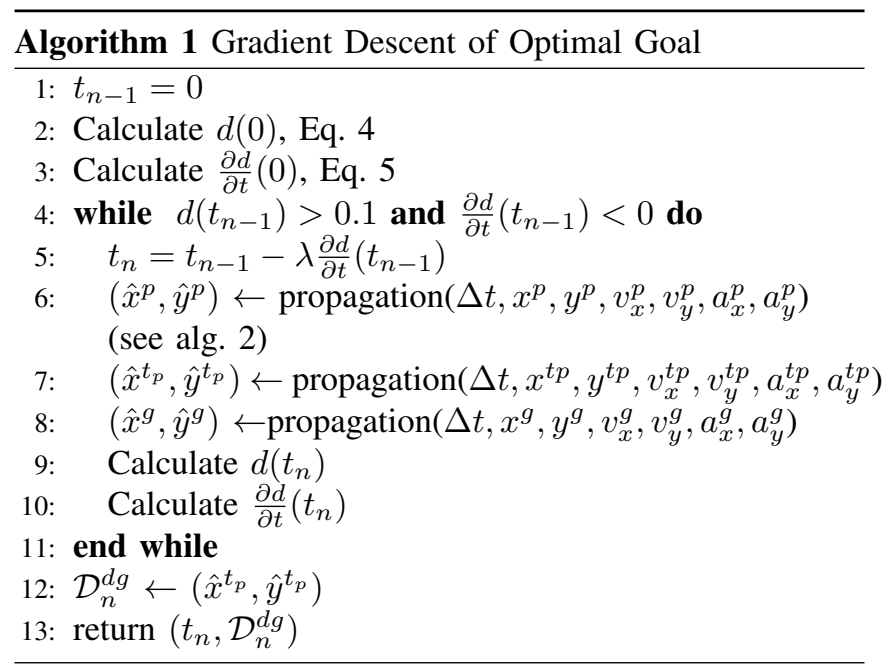

To compute the time $t_{n}$ and the dynamic goal $\mathcal{D}_{n}^{d g}, d\left(t_{n}\right)$ and $\frac{\partial d}{\partial t}\left(t_{n}\right)$ are used. They are computed as follows (we do not include $t_{n}$ to reduce the formula complexity),

$$
d=\sqrt{\left(\hat{x}^{g}-\hat{x}^{t p}\right)^{2}+\left(\hat{y}^{g}-\hat{y}^{t p}\right)^{2}}
$$

$\left(x^{g}, y^{g}\right)$ and $\left(x^{t p}, y^{t p}\right)$ are the present positions of the group and the target person, while, $\left(\hat{x}^{g}, \hat{y}^{g}\right)$ and $\left(\hat{x}^{t p}, \hat{y}^{t p}\right)$ are their predicted positions.

$$
\begin{aligned}
\frac{\partial d}{\partial t}= & \frac{\left(\hat{x}^{g}-\hat{x}^{t p}\right)\left[v_{x}^{g}+a_{x}^{g} t-v_{x}^{t p}-a_{x}^{t p} t\right]}{\sqrt{\left(\hat{x}^{g}-\hat{x}^{t p}\right)^{2}+\left(\hat{y}^{g}-\hat{y}^{t p}\right)^{2}}}+ \\
& \frac{\left(\hat{y}^{g}-\hat{y}^{t p}\right)\left[v_{y}^{g}+a_{y}^{g} t-v_{y}^{t p}-a_{y}^{t p} t\right]}{\sqrt{\left(\hat{x}^{g}-\hat{x}^{t p}\right)^{2}+\left(\hat{y}^{g}-\hat{y}^{t p}\right)^{2}}}
\end{aligned}
$$

$\left(v_{x}^{e}, v_{y}^{e}, a_{x}^{e}, a_{y}^{e}\right)$ are the velocities and accelerations of each entity, $e=\{p, t p, g\}$ person, target person or group, respectively.

Alg. 2 computes all the predictions until $t_{n}$. The predictions are computed in small time intervals, which allows to use constant speeds in the minimization problem. As we consider that all the entities have a mass $m=1$, the resulting acceleration is equal to the resulting force. Also in our case, the biggest component of the group is the robot, therefore the position of the group is propagated using the resultant force of the robot, Eq. 1. It has to be mentioned that the adaptive companion allows the group to pass one by one, this it must be ensured that at least the biggest component of the group can pass through the predicted path.

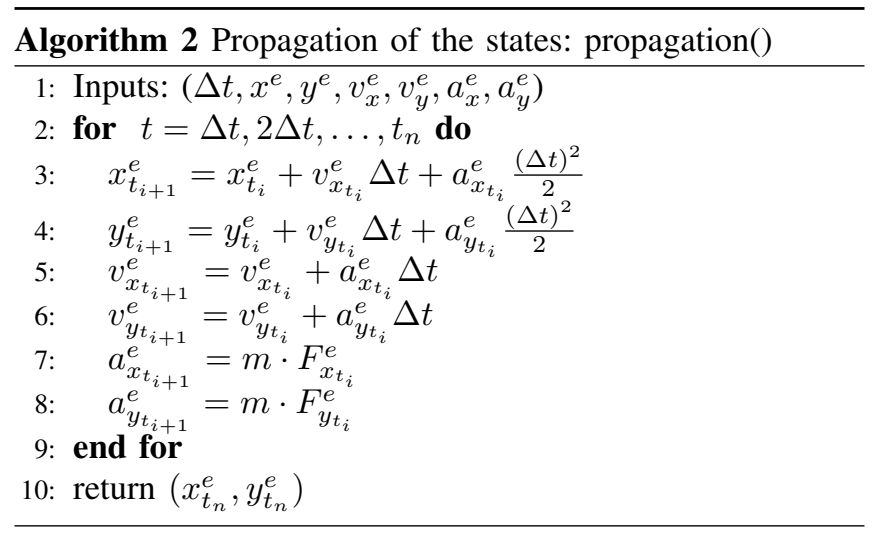

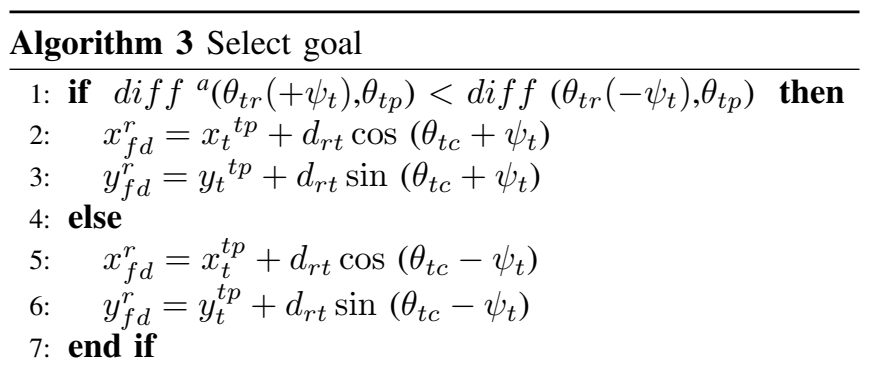

${ }^{a} \operatorname{diff}()$ is a function that calculates the difference between two orientations

2) Stage 2. Proactive configuration to create an engagement: Once the group and the target person are close, the group should face the approached person. To achieve this, we compute the angles of the triangle formed by the robot and the two humans using the cosine theorem, which uses the following equations.

$$
\begin{aligned}
& d_{t c}^{2}=d_{r c}^{2}+d_{r t}^{2}-2 d_{r c} d_{r t} \cos \left(\psi_{r}\right) \\
& d_{r c}{ }^{2}=d_{t c}^{2}+d_{r t}{ }^{2}-2 d_{t c} d_{r t} \cos \left(\psi_{t}\right) \\
& d_{r t}{ }^{2}=d_{t c}{ }^{2}+d_{r c}{ }^{2}-2 d_{t c} d_{r c} \cos \left(\psi_{c}\right)
\end{aligned}
$$

$d_{t c}$ is the distance between the target person and the companion person, $d_{r c}$ is the distance between the robot and the companion person, $d_{r t}$ is the distance between the robot and the target person, $\psi_{r}, \psi_{t}$ and $\psi_{c}$ are the angles of the vertex where the robot, the target person and the companion person are positioned.

Then, we select the robot's position using Alg. 3, which allows the robot to gaze the target person and create an engagement.

$\left(x_{f d}^{r}, y_{f d}^{r}\right)$ is robot's goal, $\left(x_{t}^{t p}, y_{t}^{t p}\right)$ is target person's position, $\theta_{t p}$ is the the target person's orientation, $\theta_{t c}$ is the orientation between the target person and the companion person, and $\theta_{t r}$ is the orientation between the target person and the robot.

\section{PERformance Metrics}

The performance metrics used to evaluate the robot behaviour are based on the proxemic rules [7].

\section{A. Performance Metrics for the Accompanying Task}

The accompanying task is evaluated using three types of performance's metrics: area, distance and angle. The values of the performances are between 0 and 1 . The best performance has value of 1 . The graphs of the performance metrics for distance and angle are described in [14].

Performance area: The best performance is achieved if the the robot is inside the area around $1 \mathrm{~m}$ from the best accompanying position [2]. Moreover, the robot gets a value of performance $1 / 2$ when its position is inside the social distances. Finally, the minimum performance is for distances far from $3 \mathrm{~m}$ or near of $0.75 \mathrm{~m}$.

Performance distance: The metric of distance has the maximum value the robot's position is inside the interval 


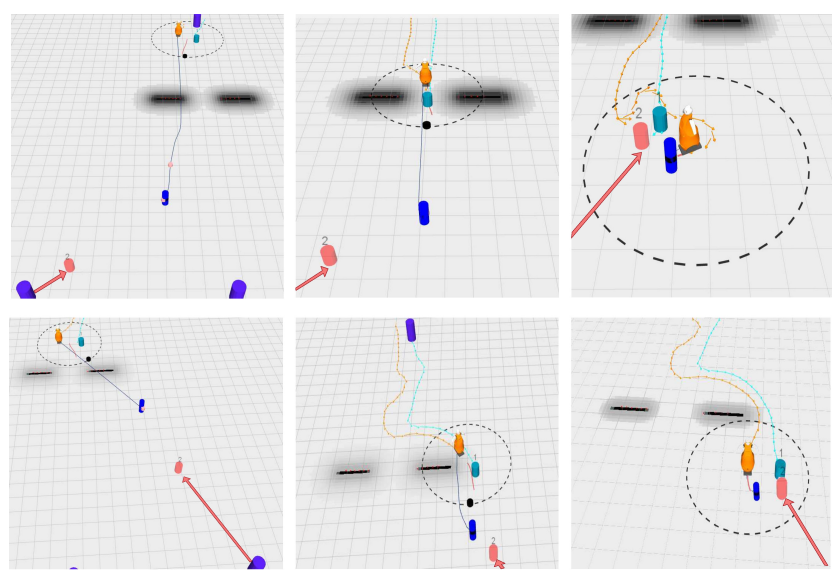

Fig. 2: Simulations. These plots represent how the group adapts its motion to obtain a more friendly encounter. In both cases the group has to avoid static obstacles.

$[1.25-2]$ meters from the accompanied person. The performance decreases if the robot approaches or moves away.

Performance angle: The metric related to the angle has value of 1 when the angle between person and robot differs as much 10 degrees from the optimal angle. The performance decreases -0.1 each time the difference between the two previous orientations increases 10 degrees.

\section{B. Performance Metrics for the Approaching Task}

To evaluate the approaching task, we verify that the distance difference between the group and the person decreases during the task or that the global path length until the dynamic goal decreases during the task.

\section{Performance Metrics for the Engagement Task}

As in the accompanying task, the final triangle formation to engage both people has three types of performance's metrics: area, distance and angle.

Performance area: It obtains the maximum value when the robot is inside the circle of $1 \mathrm{~m}$ diameter from the best robot's position respect to both people, Alg. 3. This metric follows the same rules as in the companion case.

Performance distance: It is the same as the performance distance of the accompanying case, but now the distance is between target person and robot.

Performance angle: This metric is analog to the metric of the accompanying task, but the difference of angles is between the orientation of the group and the contrary orientation of the target person.

\section{RESUlTS}

This section addresses the synthetic and real-life experiment results. Also, a user study is analyzed.

\section{A. Synthetic Experiments}

The implemented method has been tested and evaluated in a simulated dynamic environment, it uses $\mathrm{C}++$, ROS and Gazebo. This environment includes pedestrians and static obstacles. The simulated people use the ESFM to obtain
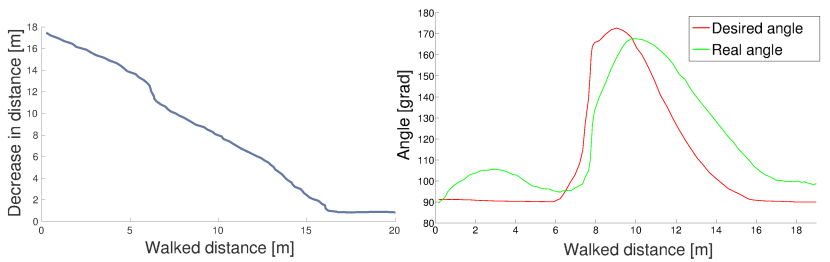

Fig. 3: Results. Left: Performance of the approaching task. Right: Desired and real angles during the accompanying task, the group has to surpass diverse people who form a corridor.

a more realistic navigation behaviour and their velocities are set randomly inside the interval $[0-1] \mathrm{m} / \mathrm{s}$. The robot uses the implemented method that combines the companion, approaching and engagement tasks. Furthermore, the accompanied person uses the AKP navigation and the approaching method, explained in this paper.

Around of 3,600 simulations were performed. In these simulations the group had to approach the target person from different directions and avoid several dynamic and static obstacles, as in the real-life experiments, see Fig. 1. An example of the simulations is shown in Fig. 2. The markers of the images are: the cyan cylinder is the accompanied person, the red cylinder is the target person, the dark blue cylinder is the dynamic goal, other pedestrians are green cylinders and static obstacles are marked in black. The black circle around the robot is the window of the local planner, the local path is marked in red and the global path until the final goal is drawn in black.

The robot behaviour combined both stages, the computation of the dynamic goal and ahieve it while accompanies his/her, and the proactive configuration to create an engagement with the target person. We evaluated the results using the metrics of performance presented in Sec. III, all results are the weighted average of all simulations.

The companion task obtained good results. It achieved a performance distance of 0.91 and a performance angle of 0.86 , a satisfactory accomplishment, and their variances were small, 0.01 and 0.015 respectively. The performance area was 0.84 and its variance was 0.0061 . Also, we got 20.32 degrees as maximum difference between the desired and the real angle, Fig 3-right. Therefore, the robot was able to perform its task properly.

Regarding the approaching task, it can be seen in Fig 3left how the distance between the group and the target person decreases during the experiment.

The results of the final positioning to do an engagement were also very good. We obtained a distance performance of 0.98 and an angle performance of 0.9 , which were close to 1 meaning a satisfactory accomplishment, and their variances were small 0.0066 and 0.015 , respectively. The area performance was 0.84 and its variance was small 0.0113 . Therefore, we can conclude that the robot performed well the engagement task. 


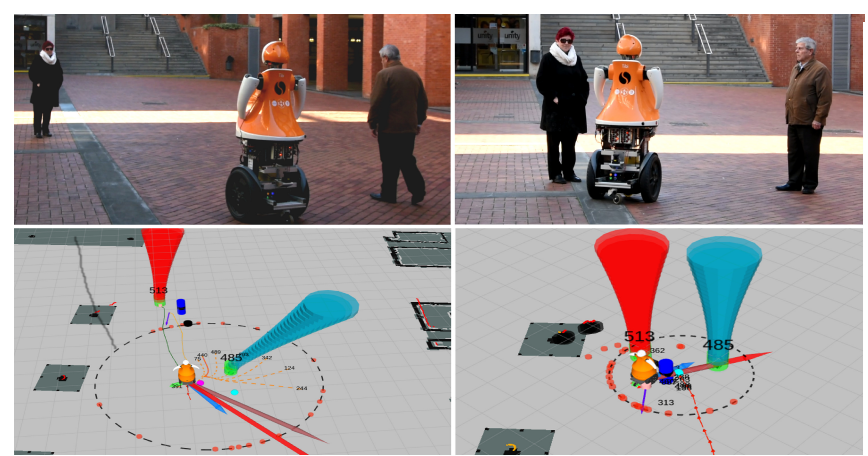

Fig. 4: User study experiments. Top: Real-life environment. Bottom: Simulated environment, representation of the Social Force Model to visualize the real-life behavior of the robot.

\section{B. Real-life Experiments}

Real-life experiments were performed using the social robot Tibi, designed to operate in urban pedestrian areas. It is equipped with multiple sensors, to detect people in the experiments we used the front and back lasers located $40 \mathrm{~cm}$ from the ground. The robot is socially accepted and humans take interest in interacting with it.

First, the method was tested in a controlled environment with pedestrians and obstacles to reproduce different types of approaching situations. The experiments were carried out in the FME (Facultat de Matemàtiques i Estadística) lab, which is a square urban area of $15 \times 15$ meters located at the South Campus of the Universitat Politècnica de Catalunya (UPC). The volunteers were technicians and students of degree, master and doctorate. No instructions were given to them.

The FME's experiments are shown in Fig. 1, where the robot was able to accompany a person, obtain the best encounter point with another person and rearrange its position to do an engagement with both people. In the images of the simulated environment we plot several markers to visualize the real-life behavior of the robot. In these pictures the green cylinders are people and their predictions are represent in the z-axis, which corresponds to time. The blue and red cylinders corresponds to the accompanied and approached person, respectively. The map and the static obstacles are painted in black. The arrows are the robot forces: in pink the force until the dynamic goal, in blue the force to maintain the formation with the accompanied person, in black and green the repulsive forces respect static obstacles and people. These forces are scaled to see them better. The circle around the robot is the window of time for the local planner.

The FME's experiments obtained good performance results, see Table I. As we explained in Sec. III, the best performance is near 1. Finally, regarding the results of the approaching task we obtained graphs where the distance between the group and the target person always decreases, as in the synthetic experiments.

Second, we performed 72 real-life experiments with different volunteers in the North campus of UPC, the Barcelona Robot Lab (BRL). A questionnaire was given to all partici- pants to carry out the user study of the Section IV-C. The 72 participants ranged in age from 15 to 76 years $(\mathrm{M}=27.21$, $\mathrm{SD}=11.72$ ), the $25 \%$ were women and $75 \%$ men, mainly students and workers of the polytechnics university campus, Fig. 4. The level of knowledge in the robotics field was included in the surveys and was ranged between 1 to 7 , the lowest level of knowledge in robotics (1,2 and 3) got 65\%, the intermediate level of knowledge in robotics (4 and 5) was around $18 \%$ and the highest level in knowledge of robotics (6 and 7)was represented by $17 \%$. Then, we can conclude that the highest amount of the participants were potential user people not related with robotics.

In each experiment, we randomly selected if the robot behaved autonomously using our method or if it was moved using tele-operation. In the experiments, Tibi was accompanying one of the participants and both encountered another person, different approach directions were performed. In addition, the final positioning was improved avoiding unnecessary movements of the robot. Now, the robot does a more human-like behavior, by positioning itself in the free space of the triangle closest to its position. After that, the robot turns towards the center of the group. This behavior assumes that both people will be turned towards the center of the group, as humans normally do.

The BRL's results of the user study for the companion and engagement tasks were also good, see Table I. Regarding the results of the approaching task we obtained similar graphs to the FME's case.

We would like to point the reader to check some videos and additional information on the following link http://www.iri.upc.edu/people/erepiso/ IROS2018.html

\section{User Study}

The results presented previously demonstrate that the robot is able to approach people while accompanies a pedestrian. A user study was also conducted to determine whether the use of the ESFM enhances the base-line model, this is, an expert moved the robot using teleop, and we should highlight that people perceived a difference between these two approaches. Social Scales: Participants were asked a set of questions following their encounter with the robot in each mode of behaviour. To analyze their responses, we grouped the survey questions into three scales: the first measured the robot's intelligence, while the second and third evaluated robot's sociability and comfortableness felt by the volunteers. Both scales surpassed the commonly used 0.72 level of reliability (Cronbach's alpha).

Each scale response was computed by averaging the results of the survey questions comprising the scale. ANOVAs were run on each scale to highlight differences between the two robot behaviours, plotted in Fig. 5. For the robot's intelligence score plotted in Fig. 5-left, pairwise comparison with Bonferroni demonstrate no statistical difference between the two kind of navigation approaches, $p>0.5$. In terms of robot's sociability and comfortableness the volunteers 


\begin{tabular}{|l|c|c|c|c|}
\hline & FME & BRL & FME & \multicolumn{1}{c|}{ BRL } \\
& \multicolumn{2}{|c|}{$\begin{array}{c}\text { Performance of Companion } \\
\text { Performance of Engagement }\end{array}$} \\
\hline Mean of Distance Performance & $0.87( \pm 0.03)$ & $0.84( \pm 0.024)$ & $0.84( \pm 0.019)$ & $0.87( \pm 0.029)$ \\
Mean of Angle Performance & $0.68( \pm 0.04)$ & $0.7( \pm 0.022)$ & $0.97( \pm 0.0025)$ & $0.84( \pm 0.022)$ \\
Mean of Area Performance & $0.73( \pm 0.067)$ & $0.65( \pm 0.039)$ & $0.84( \pm 0.017)$ & $0.83( \pm 0.025)$ \\
\hline
\end{tabular}

TABLE I: Performance results of the real-life experiments carried out in the FME and the BRL.
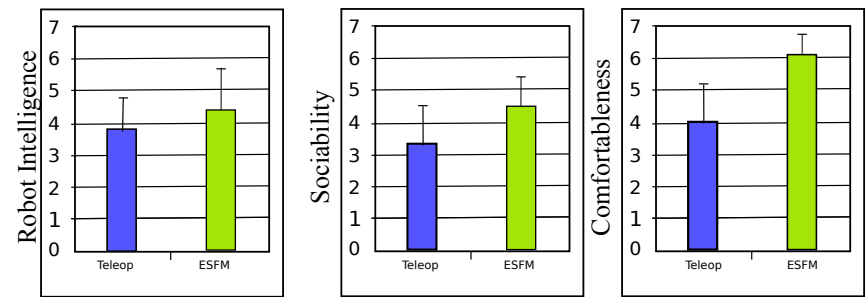

Fig. 5: User study results. Left: Robot's Intelligence. Center: Robot's Sociability. Right: Robot's Comfortableness.

perceived a difference between the two navigation's, $p<$ 0.01 in both cases.

Therefore, after analyzing these three components in navigation terms, we may conclude that if the robot has the ability to socially navigate and respect human conventions using our ESFM, it has the largest acceptance as people perceived the robot to be more sociable and comfortable.

\section{CONCLUSIONS}

This paper presents a new method to approach and engage people, while the robot performs an adaptive companion. The presented algorithm extends and improves our past work described in [1]. The major contributions of this paper are two-fold: First, we obtained a robot's behavior similar to the human's behaviour for approaching and engagement tasks. Second, the method was extensively and rigorously tested in a real-live environments in FME and in BRL environments with non-trained volunteers and a user study showed the acceptance of the method by inexpert people.

The method reformulated the ESFM to include a dynamic goal. The dynamic goal was computed using a gradient descent algorithm that allowed us to compute the optimal encounter position. Furthermore, the gradient descent method used the predictions of all people. Then, the optimal destination minimized the time, the traveled distance, the effort to accomplish the companion task and the effort to avoid obstacles and pedestrians. Furthermore, the method joined the companion, approach and engagement tasks, which was more complex, and it worked on real time. The new model was extensively tested on simulation and real-life experiments. In both cases good results were achieved.

\section{REFERENCES}

[1] E. Repiso, A. Garrell, and A. Sanfeliu, "On-line adaptive side-by-side human robot companion to approach a moving person to interact," in Iberian Robotics conference. Springer, 2017, pp. 113-125.
[2] A. Garrell and A. Sanfeliu, "Cooperative social robots to accompany groups of people," The International Journal of Robotics Research, vol. 31, no. 13, pp. 1675-1701, 2012.

[3] K. L. Koay, E. A. Sisbot, D. S. Syrdal, M. L. Walters, K. Dautenhahn, and R. Alami, "Exploratory study of a robot approaching a person in the context of handing over an object." in AAAI spring symposium: multidisciplinary collaboration for socially assistive robotics, 2007, pp. 18-24.

[4] D. Carton, A. Turnwald, D. Wollherr, and M. Buss, "Proactively approaching pedestrians with an autonomous mobile robot in urban environments," in Experimental Robotics. Springer, 2013, pp. 199214.

[5] S. Satake, T. Kanda, D. F. Glas, M. Imai, H. Ishiguro, and N. Hagita, "How to approach humans?-strategies for social robots to initiate interaction," in 4th ACM/IEEE International Conference on umanRobot Interaction. IEEE, 2009, pp. 109-116.

[6] M. Finke, K. L. Koay, K. Dautenhahn, C. L. Nehaniv, M. L. Walters, and J. Saunders, "Hey, i'm over here-how can a robot attract people's attention?" in IEEE International Workshop on Robot and Human Interactive Communication., 2005, pp. 7-12.

[7] E. T. Hall and T. Edward, "Hall. the hidden dimension," Anchor Books New York, vol. 20, p. 71, 1969.

[8] D. S. Syrdal, K. L. Koay, M. L. Walters, and K. Dautenhahn, "A personalized robot companion?-the role of individual differences on spatial preferences in hri scenarios," in The 16th IEEE International Symposium on Robot and Human interactive Communication, 2007, pp. 1143-1148.

[9] Y. Kato, T. Kanda, and H. Ishiguro, "May i help you?: Design of human-like polite approaching behavior," in Proceedings of the Tenth Annual ACM/IEEE International Conference on Human-Robot Interaction, 2015, pp. 35-42.

[10] M. L. Walters, K. Dautenhahn, R. Te Boekhorst, K. L. Koay, C. Kaouri, S. Woods, C. Nehaniv, D. Lee, and I. Werry, "The influence of subjects' personality traits on personal spatial zones in a humanrobot interaction experiment," in IEEE International Workshop on Robot and Human Interactive Communication, 2005, pp. 347-352.

[11] K. Dautenhahn, M. Walters, S. Woods, K. L. Koay, C. L. Nehaniv, A. Sisbot, R. Alami, and T. Siméon, "How may i serve you?: a robot companion approaching a seated person in a helping context," in Proceedings of the 1st ACM SIGCHI/SIGART conference on Humanrobot interaction. ACM, 2006, pp. 172-179.

[12] M. Svenstrup, S. Tranberg, H. J. Andersen, and T. Bak, "Pose estimation and adaptive robot behaviour for human-robot interaction," in EEE International Conference on Robotics and Automation, 2009, pp. 3571-3576.

[13] H.-J. Böhme, T. Wilhelm, J. Key, C. Schauer, C. Schröter, H.-M. Groß, and T. Hempel, "An approach to multi-modal human-machine interaction for intelligent service robots," Robotics and Autonomous Systems, vol. 44, no. 1, pp. 83-96, 2003.

[14] E. Repiso, G. Ferrer, and A. Sanfeliu, "On-line adaptive side-by-side human robot companion in dynamic urban environments," in IEEE/RSJ International Conference on Intelligent Robots and Systems, 2017, pp. 872-877.

[15] G. Ferrer, A. Garrell, F. Herrero, and A. Sanfeliu, "Robot socialaware navigation framework to accompany people walking side-byside," Autonomous Robots, pp. 1-19, 2016.

[16] A. Garrell, M. Villamizar, F. Moreno-Noguer, and A. Sanfeliu, "Teaching robots proactive behavior using human assistance," International Journal of Social Robotics, vol. 2, no. 9, pp. 231-249, 2017. 